\title{
Diversity of SCCmec elements in Staphylococci isolated from public washrooms
}

\author{
Hermine V. Mkrtchyan ${ }^{1}$, Zhen Xu ${ }^{1}$ and Ronald R. Cutler ${ }^{2 *}$
}

\begin{abstract}
Background: Staphylococci are commonly associated with antibiotic resistance in healthcare settings including resistance to methicillin and other beta lactam antibiotics primarily associated with the carriage of SCCmec mobile genetic elements. We investigated oxacillin susceptibility in 11 different species of environmental staphylococci to evaluate the potential dissemination of such resistance determinants in staphylococcal isolates from non-healthcare environments.

Results: Staphylococci isolated from public washrooms were analysed for SCCmec type and for antibiotic susceptibility. MICs to oxacillin ranged from 0.12 to $128 \mathrm{mg} / \mathrm{L}$. Of the 32 strains investigated, there were representatives of 11 different species of staphylococci. 21 of the 32 isolates were assigned to known SCCmec types. These typeable strains primarily included those assigned to SCCmec type I $(n=8)$, type IV $(n=6)$ and type $V I(n=7)$. In addition to these isolates, 3 strains of EMRSA-15 were identified from different environmental sites.

Conclusions: We have demonstrated the diversity of SCCmec elements in a wide range of staphylococcal species isolated from outside of healthcare settings. We have also shown that the variability in oxacillin MICs in such isolates are unrelated to species or SCCmec type. The isolation of EMRSA-15 is also of concern to infection control in the community.
\end{abstract}

Keywords: Staphylococcal species, Environmental isolates, EMRSA-15

\section{Background}

The continuing spread and development of antibiotic resistance in bacteria is recognised as a major threat to healthcare worldwide [1-3]. In the past, hospital acquired MRSA (HAMRSA) were exemplified as examples of the development and spread of drug resistance in healthcare settings however the later isolation of community acquired MRSA (CAMRSA) demonstrated that antibiotic resistant Staphylococcus aureus were also present outside of healthcare environments. Today, increasing attention is being paid to multidrug-resistant coagulase-negative staphylococci (MRCoNS) and their spread as opportunistic pathogens, often associated with infections in immunocompromised patients in healthcare settings [4-8].

Methicillin resistance is associated with the $m e c A$ gene that encodes for penicillin-binding protein PBP2a which has a low binding affinity to all $\beta$-lactam antibiotics [9]. The

\footnotetext{
*Correspondence: r.cutler@qmul.ac.uk

${ }^{2}$ Biomedical Science Degree Programmes, Pre- Clinical Drug Discovery

Group, Mile End Road, London, UK

Full list of author information is available at the end of the article
}

$m e c A$ gene is located on the staphylococcal cassette chromosome (SCCmec), a mobile genetic element with two essential components, the mec gene complex and the cassette chromosome recombinase ( $c c r)$ complex. To date, eleven (I-XI) SCCmec types have been fully identified by the determination of mec (A, B, C1, C2 and D) and $c c r$ (i.e. $c c r \mathrm{AB} 1$ to $c c r \mathrm{AB} 5$ and $c c r C$ ) complexes [10-12].

Environmental contamination with multiply drugresistant bacteria in healthcare settings is well documented $[2,6,13]$ and it has been proposed that the development of resistance in these environments is a consequence of human activities such as antibiotic misuse and/or to mutation/DNA modifying strategies in bacteria [14]. Examples of drug resistance in staphylococci isolated from non-healthcare environments have also been reported by other workers, Soge et al. [15] isolated multiply drug resistant Staphylococcus aureus (MRSA) and MRCoNS from public beaches, Coombs et al. [16] reported on regional variation with CAMRSA, Simoes et al. [17] reported that public buses were a reservoir of EMRSA-15 and ourselves, where we identified drug 
resistance in a wide variety of environmental staphylococci isolated from non-healthcare public restrooms [18].

In this current study, we report on the phenotypic expression and the diversity of SCCmec elements in oxacillin resistant determinants found in a wide range of different staphylococcal species isolated from nonhospital environments.

\section{Results and discussion}

Identification of environmental isolates and antibiotic resistance

Thirty two staphylococcal isolates with members belonging to 11 different staphylococcal species were used in this study. In these strains MICs to oxacillin varied from 0.12/0.25 mg/L to $128 \mathrm{mg} / \mathrm{L}$ (Table 1). As reported previously by other workers the MIC breakpoint to oxacillin for staphylococci is $2 \mathrm{mg} / \mathrm{L}$. However the MIC breakpoint for S.epidermidis alone has been reported to be $1 \mathrm{mg} / \mathrm{L}[19,20]$.

Table $1 \mathrm{SCC}$ ec types and Oxacillin MIC values for staphylococcal isolates

\begin{tabular}{|c|c|c|c|c|c|c|c|c|}
\hline \multirow[t]{2}{*}{ Species } & \multirow[t]{2}{*}{ No } & \multirow[t]{2}{*}{ SCCmec types } & \multicolumn{6}{|c|}{$\begin{array}{l}\text { Oxacillin MICs (mg/L) } \\
\text { (there were no isolates with } \\
\text { MICs within } 4-32 \mathrm{mg} / \mathrm{L} \text { range) }\end{array}$} \\
\hline & & & $0.12-0.25$ & 0.5 & 1 & 2 & 64 & 128 \\
\hline \multirow[t]{3}{*}{ S. hominis } & 9 & 1 & 3 & & & & & \\
\hline & & $\mathrm{Vl}$ & & & 1 & & & \\
\hline & & $\mathrm{N} / \mathrm{T}$ & 5 & & & & & \\
\hline \multirow[t]{2}{*}{ S. aureus } & 4 & IV & & & & $3^{\mathrm{a}}$ & & \\
\hline & & $\mathrm{Vl}$ & & & & & 1 & \\
\hline \multirow[t]{3}{*}{ S. warneri } & 4 & I & 1 & & & & & \\
\hline & & IV & & & 1 & & & \\
\hline & & $\mathrm{N} / \mathrm{T}$ & 2 & & & & & \\
\hline \multirow[t]{3}{*}{ S. epidermidis } & 4 & 1 & & 1 & & & & \\
\hline & & $\mathrm{Vl}$ & & & & & 1 & 1 \\
\hline & & $\mathrm{N} / \mathrm{T}$ & & & & & 1 & \\
\hline \multirow[t]{2}{*}{ S. haemolyticus } & 3 & 1 & 2 & & & & & \\
\hline & & $\mathrm{Vl}$ & & 1 & & & & \\
\hline \multirow[t]{2}{*}{ S. simulans } & 3 & IV & & 1 & & & & \\
\hline & & $\mathrm{N} / \mathrm{T}$ & 1 & & & & & 1 \\
\hline S. saprophyticus & 1 & I & 1 & & & & & \\
\hline S. equorum & 1 & $\mathrm{Vl}$ & & & & & 1 & \\
\hline S. arlettae & 1 & IV & & & & 1 & & \\
\hline S. condimeniti & 1 & $\mathrm{Vl}$ & 1 & & & & & \\
\hline S. cohnii & 1 & $\mathrm{~N} / \mathrm{T}$ & & 1 & & & & \\
\hline
\end{tabular}

Molecular characterisation of environmental staphylococcal isolates

Twenty-one out of the 32 strains tested were assigned to known SCCmec types, 11 were untypeable. The 21 typeable strains included those assigned to type I $(n=8)$, type IV $(\mathrm{n}=6)$ and type VI $(\mathrm{n}=7)$ SCCmec types (Table 2, Table 3). The Class B mec complex was detected in all isolates, including those which were untypeable. As for $c c r$ complexes, we identified $8 c c r A B 1,3 c c r A B 2$, and 7 ccrAB4 amongst these staphylococcal isolates (Table 2). SCCmec types for EMRSA-15 were determined using IVh primers (Table 3). In addition, we identified S. hominis, S. warneri, S. simulans, and S. epidermidis strains which carried untypeable $c c r$ complexes but nevertheless harboured the mecB complex.

\section{MRCoNS}

We found there was a wide diversity of SCCmec elements in a variety of different staphylococcal species isolated from non-healthcare environments, and thus diversity of SCCmec can be extensive. We found for example that the frequency of type I SCCmec elements in such staphylococcal isolates was greater than that of any of the other $\mathrm{SCCmec}$ elements. In contrast, isolates carrying SCCmec types V, VII, IX, X or XI were not found in this study. However in healthcare settings, many of the species we identified may not be investigated, although some workers have reported on specific MRCoNS from hospitals $[6,8]$. The majority of hospital strains tested were S. epidermidis and S. hominis and in these isolates SCCmec types III, IV and $\mathrm{V}$ were the most prevalent. It has also been reported, as we have found in environmental strains, that such isolates could contain multiple types of SCCmec [6].

Table $\mathbf{2}$ SCCmec types of typeable staphylococci isolates determined by combination of mec and ccr complexes

\begin{tabular}{lllll}
\hline Species & No & mec complex & ccr & SCCmec \\
\hline S. hominis & 3 & B & 1 & I \\
S epidermidis & 2 & B & 4 & $\mathrm{VI}$ \\
S. haemolyticus & 2 & B & 1 & I \\
S. arlettae & 1 & B & 2 & IV \\
S. aureus & 1 & B & 4 & $\mathrm{VI}$ \\
S. condimeniti & 1 & B & 4 & $\mathrm{VI}$ \\
S epidermidis & 1 & $\mathrm{~B}$ & 1 & $\mathrm{I}$ \\
S. equorum & 1 & $\mathrm{~B}$ & 4 & $\mathrm{VI}$ \\
S. haemolyticus & 1 & $\mathrm{~B}$ & 4 & $\mathrm{VI}$ \\
S. hominis & 1 & $\mathrm{~B}$ & 4 & $\mathrm{VI}$ \\
S. saprophyticus & 1 & $\mathrm{~B}$ & 1 & $\mathrm{I}$ \\
S. simulans & 1 & $\mathrm{~B}$ & 2 & $\mathrm{IV}$ \\
S. warneri & 1 & $\mathrm{~B}$ & 2 & $\mathrm{IV}$ \\
S.warneri & 1 & $\mathrm{~B}$ & 1 & $\mathrm{I}$ \\
\hline
\end{tabular}


Table 3 Molecular characterization of EMRSA15 from public restrooms

\begin{tabular}{lllll}
\hline Species & mecA & SCCmec & spa & MLST \\
\hline S. aureus & + & IVh & t032 & ST22 \\
S. aureus & + & IVh & t032 & ST22 \\
S. aureus & + & IVh & t032 & ST22 \\
\hline
\end{tabular}

\section{SCCmec types}

The association between the carriage of certain SCCmec types and staphylococcal species has been investigated in hospital and some SCCmec types have been reported to be associated with certain species. Type IV for example, was found to be primarily associated with hospital S. epidermidis [6]. With environmental strains however we found that SCCmec type IV was associated with S. warneri, S. simulans and S. arlettae. S. epidermidis, by contrast, was associated with SCCmec types I and VI. In addition to this, SCCmec type I, which requires a combination of mec complex B - ccrAB1, was prevalent in many staphylococci in our study. We found it in S. hominis ( $\mathrm{n}=3), S$. warneri $(\mathrm{n}=1), S$. haemolyticus $(\mathrm{n}=2), S$. saprophyticus $(\mathrm{n}=1)$ and $S$. epidermidis $(\mathrm{n}=1)$. Again, this is in contrast to previous hospital studies where it was reported that S. hominis isolated from clinical specimens was preferentially associated with ccrAB1, ccrAB4 and mec complex A [5]. These workers also described mec-ccr combinations in $S$. hominis. They identified SCCmec types I, VI, VIII and type new1, but there was a complete absence of SCCmec structures containing ccrAB2, ccrAB3, ccrC, and mec complex $\mathrm{C}$. We also failed to detect mec complex $\mathrm{C}$ in any of our isolates. The environmental isolates in our study were found to be a reservoir for mec complex B (Table 2). Bouchami and coworkers [5] proposed that the wide genetic diversity observed for coagulase negative isolates such as $S$. hominis was associated with the presence of multiple transposases and intergrases in the staphylococcal genome, this may also be the case with environmental isolates.

\section{SCCmec carriage and MICs}

Oxacillin MICs for all strains containing SCCmec types were determined. We found that the majority of coagulase negative strains (78.6\%) had relatively low MICs (0.12-1 mg/L), MICs for $S$. aureus strains could be high but still ranged from as low as $2 \mathrm{mg} / \mathrm{L}$ up to $64 \mathrm{mg} / \mathrm{L}$. In addition, there were MRCoNS strains in which the SCCmec was untypeable which produced high MIC values to oxacillin (64-128 $\mathrm{mg} / \mathrm{L})$. This was found in 2 different species, S. simulans and S. epidermidis (Table 1). Overall, comparing our results with previously published MIC breakpoints $[19,20]$, we determined that $31.25 \%$ of isolates in our study were resistant to oxacillin and $68.75 \%$ susceptible. In contrast to our findings, in a recent study of the levels of methicillin resistance in non-healthcare associated S.aureus isolated from food, the authors found that strains with MICs of $2 \mathrm{mg} / \mathrm{L}$ could be either mecA positive or mecA negative and other workers have reported that mutations in the mec complex in S.aureus can also lead to variations in MICs $[8,9]$. The wide variation in MICs we found suggests that such mutations may also occur in isolates taken from outside of hospital settings. No strains were identified with MICs in the range 4-32 $\mathrm{mg} / \mathrm{L}$.

\section{EMRSA-15 in public restrooms}

spa typing revealed that three of the MRSA strains belonged to the internationally disseminated EMRSA-15 clone with spa type t032 and MLST ST22 (Table 3). These isolates were also SCCmec subtype IVh positive. A study by Simoes and coworkers [17] demonstrated that buses, another area heavily used by the public, were also a potential reservoir for EMRSA-15. The isolates from that study and those from this study were multiply drug resistant. More than $95 \%$ of the MRSA bacteremia's in the UK are with EMRSA-15 and EMRSA-16 underscoring the importance of these isolates [17].

\section{Persistence of antibiotic resistance genes in the environment}

It is possible that a number of factors, including temperature, humidity, the presence of human faeces/ waste and the misuse of antibiotics and disinfectants (where the wrong agent is used or the agent is used at an inappropriate concentration), could contribute to the survival and spread of these environmental antibiotic resistant bacteria [14]. In addition, the mobilization of antibiotic resistant elements could also play an important role in spreading resistance genes in these environments [16].

Although only thirty two isolates were used in this study, we have shown that some well-established antibiotic resistance genes persist in the environment. This is in keeping with the ideas published in a review by Martinez in 2009 [14]. In that review it was suggested that antibiotic resistance genes originally developed in non-clinical environments. In these environments, they may have acted to control metabolic processes or for cell signalling. However, the release by man of high concentrations of antibiotics into the environment and the spread of antibiotic resistance genes has impacted on the carriage of antibiotic resistance in the environment.

Previously, we reported that public restrooms are potential reservoirs of multidrug resistant staphylococci [18]. We have now demonstrated that resistance determinants show variation between environmental isolates and commonly associated hospital isolates such as S.epidermidis and S.hominis [5, 6]. Further work is needed to establish if there are any links between 
species isolated from hospitals and those from nonclinical environments, which affect transfer of antibiotic resistance determinants. It has been suggested that environmental bacteria may respond to subclinical levels of antibiotics in the environment and thus can induce adaptive responses with low levels of resistance in some strains [21]. Many of the environmental isolates in our study possessed low (sub-clinical) levels of resistance to oxacillin $(0.12-0.25 \mathrm{mg} / \mathrm{L})$. These were even lower than the $0.5-4 \mathrm{mg} / \mathrm{L}$ MICs found in low/ borderline level oxacillin resistant clinical isolates of $S$. aureus reported by Tomasz [22].

\section{Conclusions}

The spread of low levels antibiotic resistance reported above has demonstrated the diversity of SCCmec elements in a wide range of staphylococcal species isolated from outside of healthcare settings. In addition to this, the finding of EMRSA-15 in non-healthcare environments provides further evidence that infection control measures, both in the hospitals and in public places, fails to limit the spread of such clones and once more emphasises the importance of good hygiene in these environments.

\section{Methods}

\section{Bacterial isolates and susceptibility to oxacillin}

Thirty two staphylococcal strains which had been isolated from 18 randomly selected public restrooms (non-healthcare) in London, United Kingdom [18] were used in this study. Dry sterile cotton swabs (Copan Diagnostics Inc., USA) were used to collect samples. Sampling was over a period of 24 weeks between December 2010 and June 2011. 21 sites were sampled in each restroom. All specimens were transferred to the laboratory within $1-3 \mathrm{~h}$ of the sample being taken. In the laboratory, swabs were suspended in $1 \mathrm{ml}$ sterile $0.9 \%$ saline, inoculated onto Nutrient Agar (Oxoid, Basingstoke, UK) and Mannitol Salt Agar (Oxoid, Basingstoke, UK) . Plates were incubated aerobically at $37^{\circ} \mathrm{C}$ for $24-48 \mathrm{~h}$ to obtain pure cultures.

Staphylococcal isolates were provisionally identified using conventional methods, including microscopy, catalase and coagulase testing [18]. Species were fully identified using Matrix-assisted laser desorption ionization time flight mass-spectroscopy (MALDI-TOF-MS, Microflex LT, Bruker Daltonics, Coventry, UK) [18]. Minimum Inhibitory Concentrations (MIC) to oxacillin were evaluated using "M.I.C. evaluators" (Oxoid Ltd., Basingstoke,UK). MICs were assigned on the basis of the Guidelines for Susceptibility Testing (BSAC, 2011) [19].

\section{Molecular characterization of Staphylococcal isolates SCCmec typing}

Genomic DNA was prepared using the QIAamp DNA mini kit (Qiagen, Crawley, UK). The presence of mecA was determined as described previously [18]. SCCmec types were determined by evaluating $m e c$ and $c c r$ complexes using primers previously described by others [23-26]. Type was determined using the primers J IVh F 5'-TTCCTCGTTTTTTCTGAACG-3' and J IVh R 5'-CAAACACTGATATTGTGTCG-3' as previously described [27].

SCCmec types were categorised according to guidelines published by the International Working Group on the Classification of Staphylococcal Cassette Chromosome Elements (IWG-SCC) [28] which are based on the classification of mec and ccr types. SCCmec was considered as untypeable if the primers used for PCR amplification of mec or ccr complexes did not yield amplicons.

\section{MLST and Spa typing}

S. aureus strains were analysed by Multilocus sequence typing (MLST) of seven housekeeping genes, as previously described by others [29]. Allele types were assigned using $S$. aureus MLST database (www.mlst.net). For S. aureus strains the $\mathrm{x}$-region of the protein A (spa) gene was amplified by PCR using previously described primers [30, 31]. spa types were determined using the Ridom StaphType program (Ridom GmbH, Wurzburg, Germany). Sequences for each spa gene have been deposited in the GenBank under the accession numbers KR048283, KR048284, KR048285.

\section{Abbreviations}

MRSA: Methicillin resistant Staphylococcus aureus; HAMRSA: Hospital acquired MRSA; CAMRSA: Community acquired MRSA; EMRSA: Epidemic MRSA: MRCoNS: Multi-drug resistant coagulase negative staphylococci; SCCmec: Staphylococci cassette chromosome; ccr: Cassette chromosome recombinase; MIC: Minimum inhibitory concentration; MLST: Multilocus sequence typing; PCR: Polymerase chain reaction.

\section{Competing interests}

The authors declare that they have no competing interests.

\section{Authors' contributions}

HVM participated in conceiving the study, in the design of the study, performed the molecular genetic studies, and drafted the manuscript. XZ participated in the genetic studies (SSCmec). RRC participated in conceiving the study, participated read and approved the final manuscript. All authors read and approved the final manuscript.

\section{Author details}

${ }^{1}$ Pre- Clinical Drug Discovery Group, Queen Mary University of London, School of Biological and Chemical Sciences, Mile End Road, London E1 4NS, UK. ${ }^{2}$ Biomedical Science Degree Programmes, Pre- Clinical Drug Discovery Group, Mile End Road, London, UK.

Received: 22 January 2015 Accepted: 21 May 2015

Published online: 14 June 2015

References

1. Aryee A, Price N. Antimicrobial Stewardship - can we afford to do without it? Br J Clin Pharmaco. 2014. doi:10.1111/bcp.12417. [Epub ahead of print].

2. Huttner A, Harbarth S, Carlet J, Cosgrove S, Goossens H, Holmes A, et al. Antimicrobial resistance: a global view from the World HealthcareAssociated Infections Forum. Antimicrob Resist Infect Control. 2013;2:31.

3. Yoneyama $\mathrm{H}$, Katsumata $\mathrm{R}$. Antibiotic resistance in bacteria and its future for novel antibiotic development. Biosci Biotechnol Biochem. 2006;70:1060-75. 
4. Barbier F, Ruppé E, Hernandez D, Lebeaux D, Francois P, Felix B, et al. Methicillin-Resistant Coagulase-Negative Staphylococci in the Community: High Homology of SCCmec IVa between Staphylococcus epidermidis and major clones of Methicillin-Resistant Staphylococcus aureus. J Infect Dis. 2009;202:270-81.

5. Bouchami O, Ben Hassen A, de Lencastre H, Miragaia M. Molecular Epidemiology of Methicillin-Resistant Staphylococcus hominis (MRSHo): Low clonality and reservoirs of SCCmec structural elements. PLOS One. 2011;6:e21940. doi:10.1371/journal.pOne.0021940.

6. Zong Z, Peng C, Lu X. Diversity of SCCmec elements in methicillin-resistant coagulase-negative staphylococci clinical strains. PLoS One. 2011;6:e20191. doi:10.1371/journal.pOne.0020191.

7. Shukla SK, Ramaswamy SV, Conradt J, Stemper ME, Reich R, Reed KD, et al. Novel polymorphisms in mec genes and a new mec Complex type in methicillin-resistant Staphylococcus aureus isolates obtained in rural Wisconsin. Antimicrob Agents Chemother. 2004;48(8):3080-5.

8. Bathoorn E, Hetem DJ, Alphenaar J, Kusters JG, Bonten MJ. Emergence of high-level mupiricin resistance in coagulase-negative staphylococci associated with increased short-term mupiricin use. J Clin Microbiol. 2012:50:2947-50

9. Hartman BJ, Tomasz A. Low-affinity penicillin-binding protein associated with B-lactam resistance in Staphylococcus aureus. J Bacteriol. 1984;158:513-6.

10. Matsuhashi M, Song MD, Ishino F, Wachi M, Doi M, Inoue K, et al. Molecular cloning of the gene of a penicillin-binding protein supposed to cause high resistance to $\beta$-lactam antibiotics in Staphylococcus aureus. J Bacteriol. 1986;167:975-80

11. International Working Group on the Classification of Staphylococcal Cassette Chromosome Elements (IWG-SCC). Classification of staphylococcal cassette chromosome mec (SCCmec) guidelines for reporting novel SCCmec elements-IWG-SCC. Antimicrob Agents Chemoter. 2009;53:4961-7.

12. Kondo Y, Teruyo I, Ma XX, Watanabe S, Kreiswirth BN, Etienne J, et al. Combination of multiplex PCRs for staphylococcal cassette chromosome mec type assignment: rapid identification system for mec, ccr, and major differences in junkyard regions. Antimicrob Agents Chemother. 2007:51:264-74.

13. Noble MA, Isaac-Renton $J \mathrm{~L}$, Bryce EA, Roscoe DL, Roberts FJ, Walker M, et al. The toilet as a transmission vector of vancomycin-resistant enterococci. J Hosp Infect. 1998;40:237-41.

14. Martinez JL. The role of natural environments in the evaluation of resistance traits on pathogenic bacteria. Proc R Soc B. 2009;276:2521-30.

15. Soge $\mathrm{OO}$, Meschke JS, No DB, Robetrs MC. Characterization of methicillinresistant Staphylococci aureus and methicillin-resistant coagulase-negative Staphylococcus spp. isolated from US West Coast public marine beaches. J. Antimicrob. Chemother. 2009;64:1148-55.

16. Coombs GW, Monecke S, Pearson JC, Tan H, Chew Y, Wilson L, et al. Evolution and diversity of community-associated methicillin-resistant Staphylococcus aureus in a geographical region. BMC Microbiol. 2011;11:215. doi:10.1186/1471-2180-11-215.

17. Simoes RR, Aires-de-Sousa M, Conceição T, Antunes F, Costa PM, de Lencastre H. High prevalence of EMRSA-15 in Portuguese buses: A worrisome finding. PLoS One. 2011;6:e17630. doi:10.1371/journal.pOne.0017630.

18. Mkrtchyan HV, Russell CA, Wang N, Cutler RR. Could Public restrooms be an environment for bacterial resistomes? PLoS One. 2013;8:e54223. doi:10.1371/journal.pone.00542236.

19. Andrews JM, Howe RA. BSAC standardized disc susceptibility testing method (version 10). J Antimicrob Chemother. 2011;66:2726-57.

20. Andrews JM, Boswell FJ, Wise R. Establishing MIC breakpoints for coagulase-negative staphylococci to oxacillin. J Antimicrob Chemother. 2000;45(2):259-60.

21. Aminov RI. The role of antibiotics and antibitotic resistance in nature. Environ Microbiol. 2009;11:2970-88.

22. Tomasz A, Drugeon HB, de Lencastre HM, Jabes D, McDougall L, Bille J. New mechanism for methicillin resistance in Staphylococcus aureus: clinical isolates that lack the PBP 2 a gene and contain normal penicillin-binding proteins with modified penicillin-binding capacity. Antimicrob Agents Chemother. 1989;33:1869-74.

23. Hanssen AM, Kjeldsen G, Sollid JUE. Local variants of staphylococcal cassette chromosome mec in sporadic methicillin resistant Staphylococcus aureus and methicillin-resistant coagulase-negative staphylococci: evidence of horizontal gene transfer? Antimicrob Agents Chemother. 2004:48:285-96.
24. Katayama Y, Ito T, Hiramatsu K. Genetic organization of the chromosome region surrounding mecA in clinical staphylococcal strains: Role of IS431mediated mecl deletion in expression of resistance in mecA-carrying, low-level methicillin-resistant Staphylococcus haemolyticus. Antimicrob Agents Chemother. 2001;45:1955-63.

25. Ocuma K, Iwakawa K, Turnidge JD, Grubb WB, Bell JM, O'Brien FG, et al. Dissemination of new methicillin-resistant Staphylococcus aureus clones in the community. J Clin Microbiol. 2002;40:4289-94.

26. Zhang K, McClure JA, Elsayed S, Louie T, Conly JM. Novel multiplex PCR assay for characterization and concomitant subtyping of staphylococcal cassette chromosome mec types I to V in methicillin-resistant Staphylococcus aureus. J Clin Microbiol. 2005;43:5026-33.

27. Milheirico C, Oliveira DC, de Lencastre H. Multiplex PCR strategy for subtyping the staphylococcal cassette chromosome mec IV in methicillinresistant Staphylococcus aureus: 'SCCmec IV multiplex'. J Antimicrob Chemother. 2007;60:42-8.

28. International Working Group on the Classification of Staphylococcal Cassette Chromosome Elements (IWG-SCC). Classification of Staphylococca Cassette Chromosome mec (SCCmec): Guidelines for reporting novel SCC mec elements. Antimicrob Agents Chemother. 2009;53(12):4961-7.

29. Enright MC, Day NP, Davies CE, Peacock SJ, Spratt BG. Multilocus sequence typing for characterization of methicillin resistant and methicillin-susceptible clones of Staphylococcus aureus. J Clin Microbiol. 2000;38:1008-15.

30. Harmsen D, Claus H, Witte W, Rothganger J, Claus H, Turnwald D, et al. Typing of methicillin-resistant Staphylococcus aureus in a university hospital setting by using novel software for spa repeat determination and database management. J Clin Microbiol. 2003;41:5442-8.

31. Strommenger B, Kettlitz C, Weniger T, Harmsen D, Friedrich AW, Witte W Assignment of Staphylococcus isolates to groups by spa typing, smal macrorestriction analysis, and multilocus sequence typing. J Clin Microbiol. 2006:44:2533-40

\section{Submit your next manuscript to BioMed Central and take full advantage of:}

- Convenient online submission

- Thorough peer review

- No space constraints or color figure charges

- Immediate publication on acceptance

- Inclusion in PubMed, CAS, Scopus and Google Scholar

- Research which is freely available for redistribution 